Planetary Systems in the Universe - Observation, Formation and Evolution

Proceedings IAU Symposium No. 202, (C)2004 IAU

Alan Penny, Pawel Artymowicz, Anne-Marie Lagrange, 8 Sara Russell, eds.

\title{
General-relativistic Linear Perturbation Theory on Elastical Astronomical Bodies
}

\author{
Xuejun Wu, Chongming Xu \\ Lohrmann Observatory, Technical University Dresden, Germany \\ Dept. of Phys., Nanjing Normal University, Nanjing 210097, China
}

Michael Soffel

Lohrmann Observatory, Technical University Dresden, Germany

\begin{abstract}
The general relativistic perturbations of a uniformly rotating and axisymmetric elastic deformable astronomical body in the first post-Newtonian approximation of Einstein's general relativity is discussed in a co-rotating coordinates. The main new results presented here are the post-Newtonian variations of the energy and Euler equation, which at the Newtonian level of accuracy is so called Jeffreys-Vicente equation and it play a fundamental role for the description of global geodynamics in the classical geophysics. Our results will be useful to treat the relativistic nutation and precession of the Earth and other planets.
\end{abstract}

\section{Introduction}

Pioneering works by Damour, Soffel and Xu(1991) (DSX scheme) laid the foundation for a modern theory of general relativistic celestial mechanics at the first post-Newtonian approximation of Einstein's theory of gravity. However this general relativistic DSX-formalism is not complete unless the time evolution of the (mass- and current-) multipole moments of the various astronomical bodies is completely specified(Xu, et al. 1997). In this paper we consider models of perfectly elastic (no dissipation), cold deformable astronomical bodies. Such models, at the Newtonian level, have been highly successful for the description of seismic effects and the global motion of the Earth in space (precession, nutation, polar motion, changes in the length of the day et al.). We use Carter and Quintana's formalism(1973) to discuss the perturbations of a uniformly rotating, stationary and axisymmetric astronomical body in the first post-Newtonian approximation of Einstein's theory of gravity. To describe the rotating astronomical body we profitably employ 'rotating coordinates' $\left(c \bar{T}, \bar{X}^{a}\right)$ tied to this body, which has many advantages. The metric in the rotating system will be introduced in section II. The relaxed unperturbed astronomical body, for which the shear stresses vanish everywhere and it can be described by a perfect fluid model, is defined in rotating coordinates. In Section III, as main new results we present the perturbed local evolution equations, i.e., the perturbed energy equation and the perturbed Euler equation, which at the Newtonian level of accuracy is so called Jeffreys-Vicente(1957) equation. Newtonian J-V equation is the basis to discuss the nutation and the precession of the Earth and other planets. The extended PN J-V equation is the first time deduced by ourselves. 


\section{The Unperturbed Metric in Rotating Coordinates}

Accordance to the notation used in DSX scheme, the local coordinates is denoted by capital letters, i.e., by $X^{\alpha}=\left(c T, X^{a}\right)$. We introduce a rotating coordinates $\bar{X}^{\alpha}=\left(c \bar{T}, \bar{X}^{a}\right)$, which satisfies the coordinate transformation $\bar{T}=T$, $\bar{X}^{a}=R^{a b} X^{b}$, where $R^{a b}(T)$ is a time-dependent rotation matrix, $d R^{a b} / d T=$ $\epsilon_{b c d} \Omega^{c} R^{a d}$. From this we can easily derive the metric tensor in rotating coordinates

$$
\begin{aligned}
\bar{G}_{00} & =-\exp \left(-\frac{2 \bar{W}+\bar{V}^{2}}{c^{2}}\right)+\mathcal{O}(5), \\
\bar{G}_{0 a} & =\frac{\bar{V}^{a}}{c}-\frac{4 \bar{W}_{a}}{c^{3}}+\mathcal{O}(5), \\
\bar{G}_{a b} & =\delta_{a b} \exp \left(\frac{2 \bar{W}}{c^{2}}\right)+\mathcal{O}(4)=\delta_{a b}\left(1+\frac{2 \bar{W}}{c^{2}}\right)+\mathcal{O}(4),
\end{aligned}
$$

where we use the notation $\mathcal{O}(n) \equiv \mathcal{O}\left(c^{-n}\right) . \bar{W} \equiv W+\frac{2 W V^{2}}{c^{2}}-\frac{4 W^{b} V^{b}}{c^{2}}+\frac{V^{4}}{4 c^{2}}, \bar{W}_{a} \equiv$ $R^{a b}\left(W_{b}-\frac{1}{2} V^{b} W\right) . W$ and $W_{a}$ are the scalar- and vector-potential that completely determine the metric to first post-Newtonian order in non rotating coordinates.

\section{The Perturbed Configuration}

In order to describe small deviations from the relaxed ground state of our astronomical body we will employ the formalism devised by Carter and Quintana. The Eulerian variations of the energy-momentum tensor $\delta T_{\mu \nu}$ can be taken as

$$
\delta T_{\mu \nu}=\delta \rho u_{\mu} u_{\nu}+\delta p \gamma_{\mu \nu}+\left(\rho c^{2}+p\right) \delta \gamma_{\mu \nu}-\rho c^{2} h_{\mu \nu}-2 \mu s_{\mu \nu},
$$

where $u_{\mu}$ is 4-velocity, $\gamma_{\mu \nu}=g_{\mu \nu}+u_{\mu} u_{\nu} / c^{2}$ is the projection tensor, $h_{\mu \nu}=\delta g_{\mu \nu}$ is the Eulerian variation of metric $g_{\mu \nu}, s_{\mu \nu}$ is the shear tensor. For an unsheared reference state $\delta s_{\mu \nu}=s_{\mu \nu}$. The covariant displacement field, for obvious reasons, will be defined in rotating coordinates. It is taken as $\xi^{\alpha}=\left(0, s^{a}\right)$, since from adjusting the initial time and gauge it is always possible to define $\xi^{0}=0$. The covariant components are then given by $\xi_{\alpha}=\left(\bar{G}_{0 a} s^{a}, \bar{G}_{b a} s^{a}\right)$. To postNewtonian accuracy and first order in the displacement field we can derive the relations between the displacement field and the shear tensor

$$
\begin{aligned}
s_{00} & =\mathcal{O}(4), \quad s_{0 a}=\mathcal{O}(5) \\
s_{a b} & =s^{(a}{ }_{, b)}-\frac{1}{3} \delta_{a b} s^{c}{ }_{, c}+\frac{1}{c^{2}}\left[2 \bar{W}\left(s^{(a}{ }_{, b)}-\frac{1}{3} \delta_{a b} s^{c}{ }_{, c}\right)+\bar{V}^{c} s^{c}{ }_{, a} \bar{V}^{b)}+\bar{V}^{(a} \dot{s}^{b)}\right. \\
& \left.+\bar{V}^{(a} \epsilon_{b) c d} \bar{\Omega}^{c} s^{d}-\frac{1}{3} \delta_{a b} \bar{V}^{c} \dot{s}^{c}-\frac{1}{3} \bar{V}^{a} \bar{V}^{b} s^{c}{ }_{, c}-\frac{1}{3} \delta_{a b} \bar{V}^{c} \epsilon_{c d e} \bar{\Omega}^{d} s^{e}\right]+\mathcal{O}(4),(6)
\end{aligned}
$$

where $\dot{s}^{b} \equiv s_{\bar{T}}^{b}$ and two indices enclosed in round brackets imply symmetrization. The Eulerian variation of the pressure is

$$
\delta p=-\rho^{*} \bar{W}_{\mathrm{G}, b} s^{b}-\kappa \Theta+\frac{\kappa}{c^{2}}\left(4 \bar{W} \Theta+\bar{V}^{a} \bar{V}^{b} s^{a}, b\right)+\mathcal{O}(4),
$$


where the volume dilatation $\Theta$ is given by

$$
\Theta \equiv e_{\mu}^{\mu}=s_{, b}^{b}+\frac{1}{c^{2}}\left(\bar{V}^{b} \dot{s}^{b}+\epsilon_{b c d} \bar{\Omega}^{c} s^{d} \bar{V}^{b}+3 \bar{W}_{, c} s^{c}+3 \delta \bar{W}\right)+\mathcal{O}(4) .
$$

The main results of this paper concern the perturbed local evolution equations $\delta\left(\bar{T}_{\mu ; \nu}^{\nu}\right)=0$ in the rotating coordinate system. Taking $\mu=0$ and $\mu=a$ we obtain the perturbed energy balance equation and the perturbed Euler equation as

$$
\begin{aligned}
\delta \rho= & -\nabla \cdot(\rho \mathbf{s})-\frac{1}{c^{2}}\left(\rho \bar{V}^{a} \dot{s}^{a}+\left(p s^{a}\right)_{, a}+2 \bar{W}_{, a} s^{a} \rho+3 \rho \delta \bar{W}\right)+\mathcal{O}(4) \\
= & -\rho_{, a} s^{a}-\rho^{*} \Theta+\mathcal{O}(4), \\
0= & \rho^{*}\left(1+\frac{2 \bar{W}_{\mathrm{G}}}{c^{2}}\right)\left(\ddot{s}_{a}+2 \epsilon_{a b c} \bar{\Omega}^{b} \dot{s}^{c}\right)+\rho^{*} \Theta \bar{W}_{\mathrm{G}, a}-\rho^{*} s_{, a}^{b} \bar{W}_{\mathrm{G}, b}-\rho^{*}\left(\delta \bar{W}_{\mathrm{G}}\right)_{, a} \\
& -\rho^{*} s^{b} \bar{W}_{\mathrm{G}, b a}-\left(\kappa \Theta \delta_{a \beta}+2 \mu s^{\beta}{ }_{a}\right)_{; \beta}+\frac{1}{c^{2}}\left\{\rho ^ { * } \left[\bar{V}^{a}\left(\bar{V}^{b} \ddot{s}^{b}\right)+\bar{W}_{\mathrm{G}, b} \dot{s}^{b} \bar{V}^{a}\right.\right. \\
& \left.-2 \bar{V}^{b} \dot{s}^{b} \bar{W}_{\mathrm{G}, a}+(\delta \bar{W})_{, \bar{T}} \bar{V}^{a}+8 \dot{s}^{b} \bar{W}_{[b, a]}-4\left(\delta \bar{W}_{a}\right)_{, \bar{T}}\right] \\
& \left.+\kappa\left(\Theta \bar{W}_{\mathrm{G}, a}-\dot{\Theta} \bar{V}^{a}\right)+\left(\kappa\left(4 \bar{W} \Theta+\bar{V}^{b} \bar{V}^{c} s_{, c}^{b}\right)\right)_{, a}\right\}+\mathcal{O}(4),
\end{aligned}
$$

where $\rho^{*} \equiv \rho+p / c^{2}$ and $\bar{W}_{G} \equiv \bar{W}+\bar{V}^{2} / 2$. Note that the part containing the shear-stress tensor reads explicitly

$$
\begin{aligned}
\left(2 \mu s^{\beta}{ }_{a}\right)_{; \beta}= & \left(2 \mu s_{b a}\right)_{, b}+\frac{1}{c^{2}}\left\{-\left(4 \mu \bar{W} s_{b a}+2 \mu s_{c a} \bar{V}^{c} \bar{V}^{b}\right)_{, b}+\left(2 \mu s_{a b}\right)_{, \bar{T}} \bar{V}^{b}\right. \\
& \left.+2 \mu\left(2 \bar{W}_{, c} s_{a c}+\epsilon_{a c b} \Omega^{c} s_{b d} \bar{V}^{d}\right)\right\} .
\end{aligned}
$$

This is the desired dynamical equation for the displacement field. It is valid up to terms of $\mathcal{O}(4)$ and second order in the displacement field itself.

Acknowledgments. This work was supported by the Deutsche Forschungs Gemeinschaft (DFG). C. X. \& X. W. would thank also the Chinese National Natural Science Foundation(No.19873008 and 19835040).

\section{References}

Carter, B. 1973, Commun. Math. Phys., 30, 261

Damour, T., Soffel M., \& Xu, C. 1991, Phys.Rev.D, 43, 3273

Jeffreys, H., \& Vicente, R. O. 1957, MNRAS, 117, 142

Xu, C., Wu, X., \& Schäfer, G. 1997, Phys.Rev.D, 55, 528 